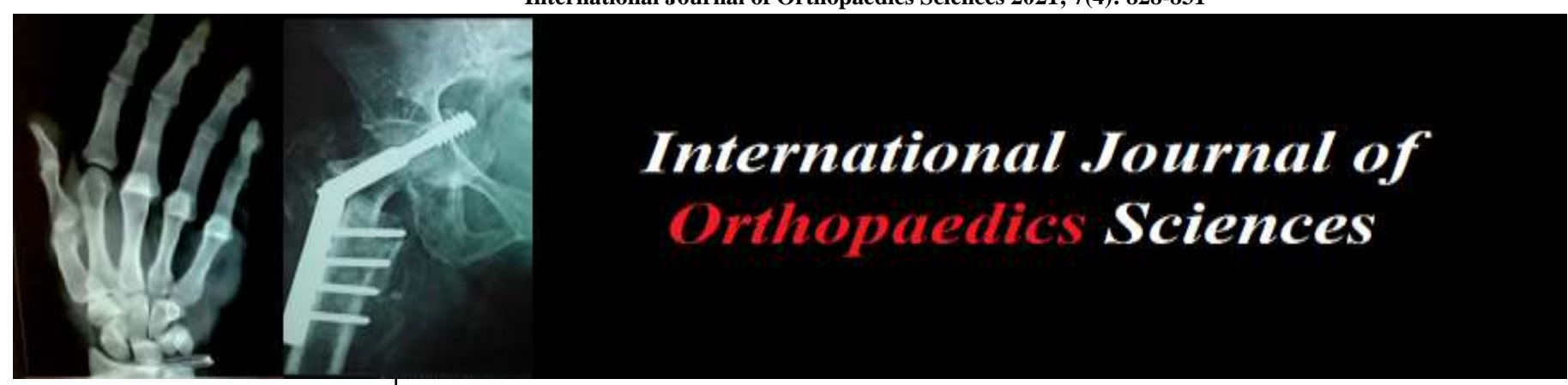

E-ISSN: 2395-1958

P-ISSN: 2706-6630

IJOS 2021; 7(4): 828-831

(C) 2021 IJOS

www.orthopaper.com

Received: 02-08-2021

Accepted: 13-09-2021

Dr. P Sathyamurthy

PSG Institute of Medical

Sciences and Research

Coimbatore, Tamil Nadu, India

Dr. Udayamoorthy

PSG Institute of Medica

Sciences and Research,

Coimbatore, Tamil Nadu, India

Dr. Prabharam Kumar

PSG Institute of Medical

Sciences and Research,

Coimbatore, Tamil Nadu, India

Dr. Aakash Kannauh

PSG Institute of Medical

Sciences and Research,

Coimbatore, Tamil Nadu, India

\section{Novel method of intraoperative sign language to minimize cross infection of COVID-19 in trauma operating rooms}

\author{
Dr. P Sathyamurthy, Dr. Udayamoorthy, Dr. Prabharam Kumar and Dr. \\ Aakash Kannauh
}

DOI: $\underline{\text { https://doi.org/10.22271/ortho.2021.v7.i41.2974 }}$

\section{Abstract}

Introduction and Purpose: COVID-19 pandemic has changed the field of traumatology world-wide. Exposure by inhalation of droplets from an infected person is the main route of spread. Numerous studies has shown aerosols are generated not only by coughing and sneezing but also by speaking, talking loudly and whisper. Lot of talking is involved during trauma surgery during handling instruments. Mask wearing especially N95 can force us to use louder speech. Here we introduce a new universal sign language for orthopaedic instruments and maneuvers inside the operating rooms in order to reduce the speech involved so that inter personnel spread can be minimized.

Materials and Methods: We have charted a universal hand gestures for most commonly used orthopaedic instruments and maneuvers commonly involved in a fracture fixation surgery. These gestures are taught to all doctors and staffs involved in fracture surgery. The staffs are motivated to use gestures instead of speaking. Each instruments when needed is indicated by a specific gesture. For quick reference a simplified chart showing the gestures can be pasted inside operating room walls.

Conclusion: Operating a COVID positive patient during his infectious period is inevitable since trauma surgeries cannot be delayed unlike elective surgeries. Avoiding excessive talking during the procedure and using sign languages inside operating rooms can minimize viral load from operating personnel and therefore can safeguard medical professionals.

Keywords: Transverse patella fracture, tension band wiring, functional outcome

\section{Introduction}

As of late March 2020, the global COVID-19 pandemic caused by the SARS-CoV-2 virus has battered the world. Financial markets have crashed, restaurants and public places are deserted; countries have effectively closed their borders; and millions of people are confined to their homes under shelter-in-place orders. Virologists and epidemiologists are racing to understand COVID-19 and how best to treat it. Many unknowns remain, but one thing is eminently clear: COVID-19 is both deadly and highly transmissible.

\section{Effect of speech in the transmission}

A mysterious aspect, however, involves how it is so transmissible? The proposed mechanism is by direct or indirect contact of the virus containing aerosol. These aerosols are liberated at an enormous amount during the process of Coughing or sneezing. It is less widely known that normal speaking also produces thousands of oral fluid droplets with a broad size distribution. A substantial number of studies have, however, investigated aerosols emitted from breathing, talking, coughing and sneezing (e.g. Asadi et al. 2019; Johnson et al. 2011) ${ }^{[1,2]}$. It has also been shown that just normal breathing over time can generate more viable virus aerosol than coughing, since the latter is a less frequent activity (Lindsley et al. 2016) ${ }^{[3]}$.

\section{COVID and orthopaedic surgery}

The worldwide COVID-19 pandemic is directly impacting the field of orthopaedic surgery and traumatology with postponed operations, changed status of planned elective surgeries and acute emergencies in patients with unknown infection status.
Dr. P Sathyamurthy PSG Institute of Medical Sciences and Research, Coimbatore, Tamil Nadu, India 
With globally increasing numbers as of late March 2020, the global COVID-19 pandemic caused by the SARS-CoV-2 virus has battered the world. Financial markets have crashed, restaurants and public places are deserted; countries have effectively closed their borders; and millions of people are confined to their homes under shelter-in-place orders. Virologists and epidemiologists are racing to understand COVID-19 and how best to treat it. Many unknowns remain, but one thing is eminently clear: COVID-19 is both deadly and highly transmissible.

\section{Effect of speech in the transmission}

A mysterious aspect, however, involves how it is so transmissible? The proposed mechanism is by direct or indirect contact of the virus containing aerosol. These aerosols are liberated at an enormous amount during the process of Coughing or sneezing. It is less widely known that normal speaking also produces thousands of oral fluid droplets with a broad size distribution. A substantial number of studies have, however, investigated aerosols emitted from breathing, talking, coughing and sneezing (e.g. Asadi et al. 2019; Johnson et al. 2011) ${ }^{[1,2]}$. It has also been shown that just normal breathing over time can generate more viable virus aerosol than coughing, since the latter is a less frequent activity (Lindsley et al. 2016) ${ }^{[3]}$.

\section{COVID and orthopaedic surgery}

The worldwide COVID-19 pandemic is directly impacting the field of orthopaedic surgery and traumatology with postponed operations, changed status of planned elective surgeries and acute emergencies in patients with unknown infection status. With globally increasing numbers of infected patients and the resulting restrictions on the elective surgical care of musculoskeletal patients, orthopaedic surgeons are faced with challenges in providing continuity of care to musculoskeletal patients, while ensuring the safety of patients and hospital personnel.

But operating a COVID patient with trauma is inevitable. Health professionals are exposed to the virus not only from the patient, but also from each other. This is because health care workers are constantly encountering many in and out patients with COVID status unclear. When they are clubbed inside a compact air conditioned room the risk of cross infection becomes high.

\section{Talking during trauma surgeries}

During surgeries, especially while operating an orthopaedic trauma or fracture patients the need for speaking and talking increases. The increased need for talking is attributed to use of number of instruments in orthopaedic surgeries compared to other surgical departments.

The wearing of mask or PPE increases the need for increased loudness and intensity. Especially wearing double masks, N95 masks or personal protective respirators are mandatory in COVID times. According to Elena et al. the effort needed to speak increases with increased thickness of mask used ${ }^{[4]}$. According to Patricia et al. opening of the mouth and exposing the mucous membrane will increase the exposure to aerosol and thereby leading to increased transmission. ${ }^{5}$ According to that study primary surgeon and first assistants are the most vulnerable.

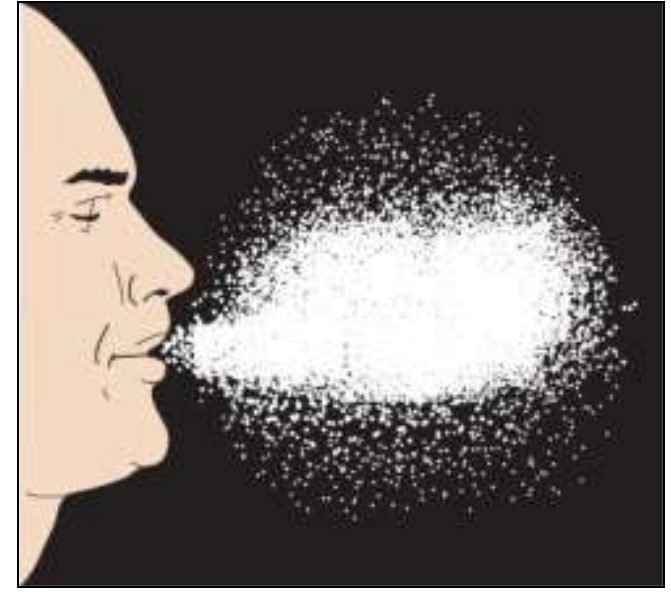

Fig 1: Aerosols while speaking violently

\section{Need For Intra-operative Sign language}

In order to minimize the speaking inside operating rooms and thereby decrease the COVID cross infection, a need for universal sign language using gestures and hand signs come into play.

Sign languages are not new to medical field. First physician to use sign language is Dr. Jean Mark Gaspar Itard in early 1800. These kind of sign languages are available for explaining to deaf patients by variety of methods.

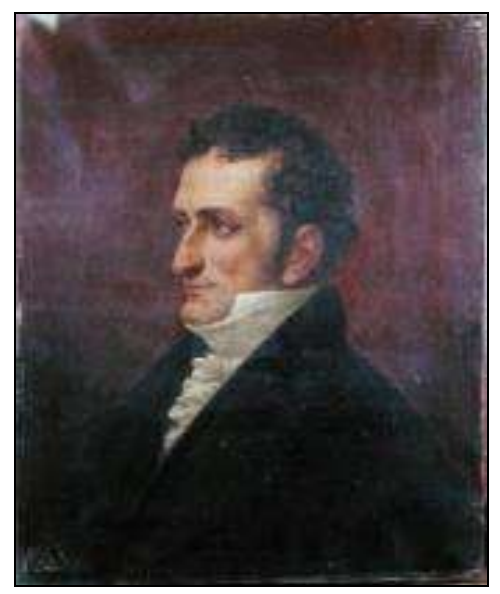

Fig 2: Dr. Jean Mark Gaspar Itard

But these are limited use and not applicable to surgical instruments and implants. So we have designed a universal sign language for orthopaedic instruments and implants commonly used in fracture surgeries. Arthroplasty and arthroscopy instruments are not included in this study because they are usually elective procedures.

\section{Materials and methods}

The common instruments and implants used in a fracture fixation case are listed. These included both basic surgical equipments (scalpel, scissors, artery forceps, tissue retractors etc), orthopaedic fracture equipments (bone holder, plate holding forceps, point reduction clamps, reamers, guide wires, entry awl, screw drivers etc), orthopaedic implants (intramedullary nails, bone plates, screws, cerclage wires, kwires etc), maneuvers (mopping, retracting, traction, suctioning, C-arm firing etc). They are tabulated according to their types. 
Each item is assigned a specific number of two digits. The first number indicates the type of the item (general, reduction, implant or maneuver) the second digit indicates the specific item. Each items are assigned a specific hand gesture. The hand gestures are usually assigned based on the use of that item or mechanism /mode of usage of that particular item.

Exclusion criteria: Unusual instruments and rarely used items are excluded. Arthroplasty, arthroscopy and spine instruments are excluded.

\section{First digit}

Table 1: Shows in First digit for reference

\begin{tabular}{|c|c|}
\hline Type of the item & First digit for reference \\
\hline General surgical & 1 \\
\hline Fracture allied & 2 \\
\hline Maneuvers & 3 \\
\hline Implants & 4 \\
\hline
\end{tabular}

Table 2: Shows in 2 digit No

\begin{tabular}{|c|c|c|c|c|c|c|c|}
\hline $\begin{array}{c}\text { General } \\
\text { Instrument }\end{array}$ & $\begin{array}{l}2 \text { digit } \\
\text { No }\end{array}$ & $\begin{array}{c}\text { Fracture allied } \\
\text { instruments }\end{array}$ & $\begin{array}{l}2 \text { digit } \\
\text { No }\end{array}$ & Maneuver & $\begin{array}{l}2 \text { digit } \\
\text { No }\end{array}$ & Implants & $\begin{array}{c}2 \text { digit } \\
\text { No }\end{array}$ \\
\hline Scalpel & 1.1 & Bone holder & 2.1 & Suctioning & 3.1 & $\mathrm{~K}$ wire & 4.1 \\
\hline Scissors & 1.2 & Plate holder & 2.2 & Mopping the field & 3.2 & Nail & 4.2 \\
\hline L Retractors & 1.3 & Point reduction forceps & 2.3 & Gauze piece & 3.3 & Plate & 4.3 \\
\hline Forceps & 1.4 & Periosteal elevator & 2.4 & Traction & 3.4 & Screw & 4.4 \\
\hline Hemostat curved & 1.5 & Hohmanns retractor & 2.5 & $\mathrm{C}$ arm firing & 3.5 & SS wire & 4.5 \\
\hline Hemostat straight & 1.6 & Awl & 2.6 & Retracting & 3.6 & Schanz pin & 4.6 \\
\hline Diathermy & 1.7 & guide wire & 2.7 & Rotating the limb & 3.7 & Steinmann pin & 4.7 \\
\hline Suture & 1.8 & reamer & 2.8 & Wash & 3.8 & Exfix rod & 4.8 \\
\hline Needle holder & 1.9 & Screw driver & 2.9 & Drill & 3.9 & Exfix clamp & 4.9 \\
\hline
\end{tabular}

Each items are also assigned with a special unique hand gesture using both hands and fingers. These gestures and number reference chart are printed out and pasted in operating rooms and also given to all the personnel involved in the surgery including staff nurses. They are asked to memorize the number and codes. During surgery the primary surgeon will ask for specific item or maneuver using the hand gestures first. A few examples are shown below.
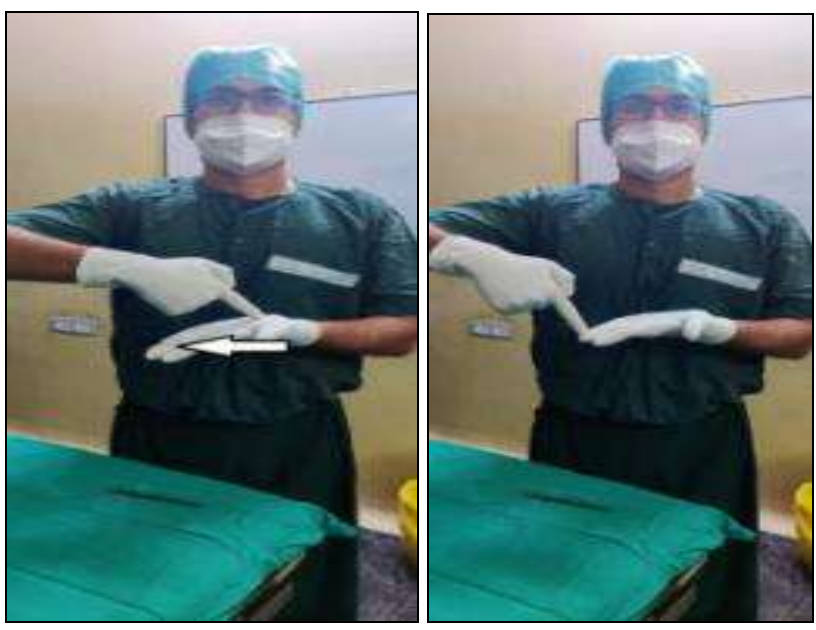

Fig 3: Scalpel running index finger over palm proximal to distal. (Code -1.1)

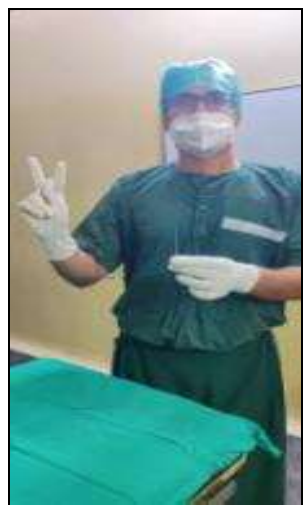

Fig 4: Gesture for $\mathrm{K}$ wire - thumb at the base of $2^{\text {nd }}$ and $3^{\text {rd }}$ finger making a ' $\mathrm{V}$ ' (code- 4.1)

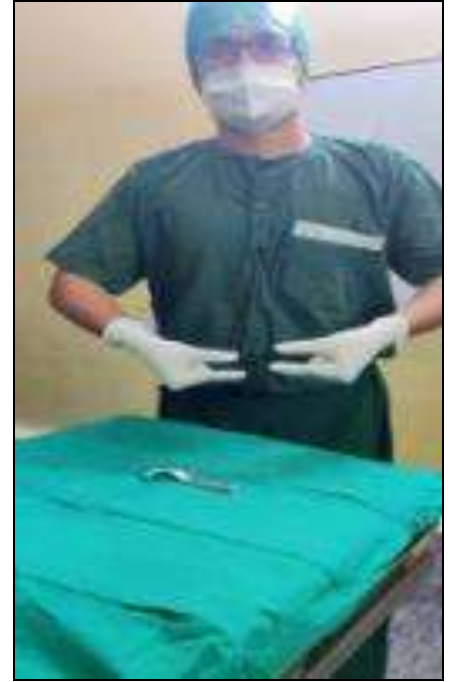

Fig 5: Bone plate - indicated by pinching of index and thumb of both hands without touching. (Code- 4.3)
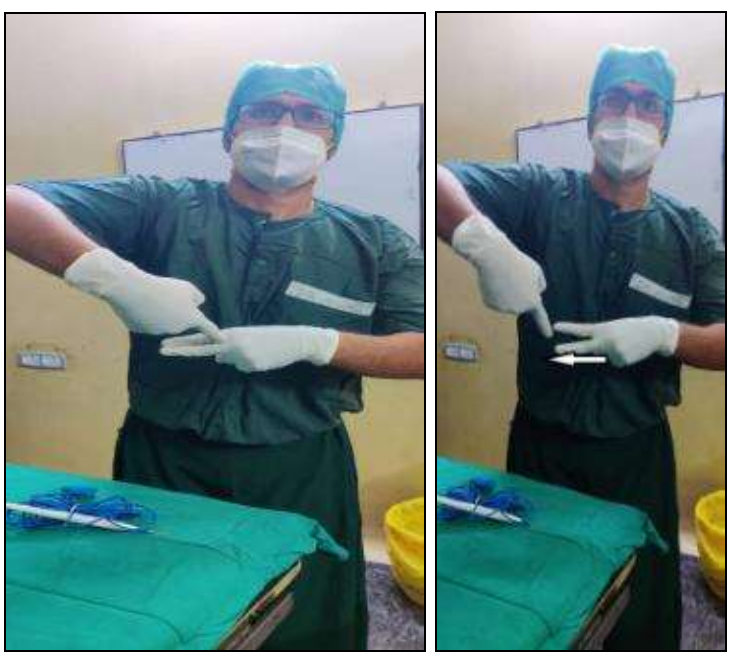

Fig 6: Diathermy indicated by running index finger of one hand between 2 and $3^{\text {rd }}$ finger of opposite hand and making a gesture of opening the digits. (Code 1.7)

If the assistant is not able to understand the item the assistant will cross his hands like below. 


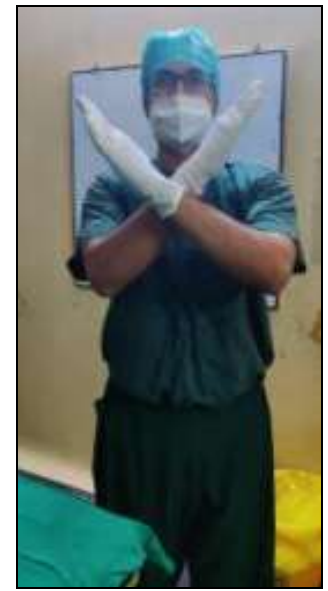

Fig 7: Crossing limbs to indicate "I didn't Understand"

If the staff/assistant not able to understand the two-digit number code of the specific instrument is used. first number flashed with fingers will indicate type of the item (general/fracture allied/maneuver/implant).

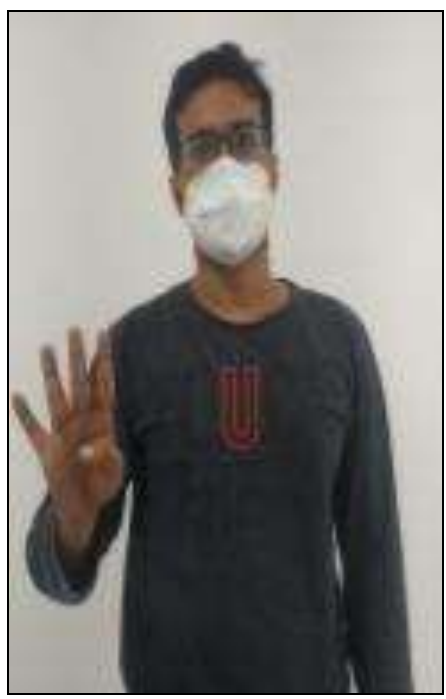

Fig 8: $1^{\text {st }}$ digit flashing (4)

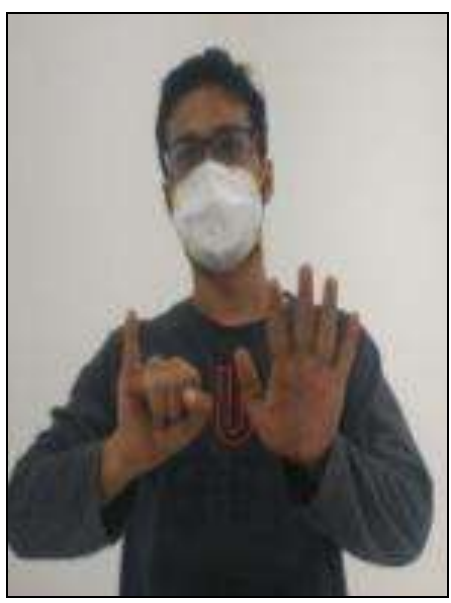

Fig 9: $2^{\text {nd }}$ digit flashing (indicates 6)

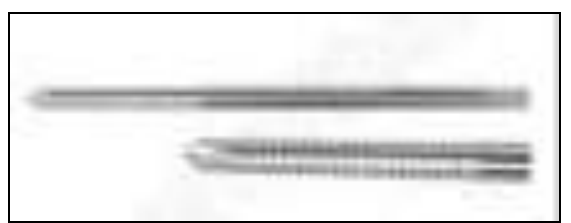

Fig 10: The above indicates 4.6 that means schanz pin

\section{Discussion}

We implemented this system for nearly 15 patients who were teated for fractures operatively who were tested positive for COVID virus. Though initially we found difficult in using the sign language as time went on all OT personnel got accustomed to it. We encourage them to reduce speaking and to speak only when it is absolutely necessary.

One may think why to use two methods (gesture and numbering). It was easier to use because we had two methods for indicating a specific item. Even when one method fails we can immediately opt for the second one. And numbering system was a bit easier to understand and remember.

The draw backs of this method is it doesn't include all the instruments and it doesn't specify the size of the specific item. For example, a periosteal elevator can be small, medium or large and need a specific gesture to identify each which will be improved in near future. As far as bone plates and nails are concerned specific sizes need to be said orally only and need specific gesture or number. We are trying to add more and more instruments. Arthroplasty and arthroscopic equipments are not included and needs to be done. Repeated changing of staffs will face difficulties to get accustomed. So we advise to better not to change OT staffs frequently. Whenever we faced difficulty in gestures we opted for verbal communication using simple and less number of words. The surgeons usually will get exhausted after prolonged surgeries. This method may help to minimize it too.

\section{Conclusion}

We are in a phase of cruel pandemic. We, orthopaedic surgeons need to evolve to cope up with the present condition. By using this method, there is a chance for lesser cross contamination, however low it may be. It not only reduces COVID cross infection, but it helps to reduce surgical site infections also as proven by previous studies. This method can be used as an adjunct to wearing masks, PPE and other equipments. Accepting this method universally can minimize confusion due to various methods. Overall, adopting this method will be very helpful in this COVID crisis.

\section{References}

1. Asadi S, Wexler AS, Cappa CD, Barreda S, Bouvier NM, Ristenpart WD. Aerosol emission and superemission during human speech increase with voice loudness. Sci Rep. 2019;9:2348.

2. Johnson, Morawska GRL, Ristovski ZD, Hargreaves M, Mengersen K, Chao CYH. et al. Modality of human expired aerosol size distributions. Journal of Aerosol Science. 2011;42:839-51.

3. Lindsley WG, Blachere FM, Beezhold DH, Thewlis RE, Noorbakhsh B, Othumpangat S et al. Viable influenza a virus in airborne particles expelled during coughs versus exhalations. Influenza Other Resp. 2016;10:404-13.

4. Giovanelli E. Unmasking the difficulty of listening to talkers with mask: lessons from COVID pandemic. [Internet]; Available from: https://orcid.org/0000-00017753-632X; DOI: 10.1177/2041669521998393

5. Heinsohn P, Jewett DL. Exposure to blood containing aerosols in the operating room: a preliminary study, American Industrial Hygiene Association Journal. 1993;54:8. 